\title{
Evidence of the neuroprotective role of citicoline in glaucoma patients
}

\author{
Vincenzo Parisi ${ }^{1, *}$, Giovanni Coppola ${ }^{2}$, Marco Centofanti ${ }^{1,3}$, Francesco Oddone $^{1}$, \\ Anna Maria Angrisani ${ }^{2}$, Lucia Ziccardi ${ }^{1}$, Benedetto Ricci ${ }^{2}$, Luciano Quaranta $^{4}$ and \\ Gianluca Manni ${ }^{1,3}$ \\ ${ }^{1}$ G. B. Bietti Eye Foundation-IRCCS, Rome, Italy \\ ${ }^{2}$ Ophthalmology Unit, Association Columbus Clinic, Catholic University of Rome, Rome, Italy \\ ${ }^{3}$ Department of Ophthalmology, University of Rome Tor Vergata, Rome, Italy \\ ${ }^{4}$ Department of Ophthalmology, University of Brescia, Brescia, Italy
}

\begin{abstract}
The glaucomatous disease is currently considered a disease involving ocular and visual brain structures. This new approach to glaucoma introduces the possibility of inducing an improvement by means of a pharmacological approach similar to that used in different degenerative brain disorders. In line with this hypothesis, we studied the effects of oral $\left(1600 \mathrm{mg} / \mathrm{die}\right.$, Cebrolux ${ }^{\mathbb{R}}$, Tubilux Pharma, Pomezia, Rome, Italy) or intramuscular $\left(1000 \mathrm{mg} / \mathrm{die}\right.$, Cebroton ${ }^{\circledR}$, Tubilux Pharma) cytidine-5'-diphosphocholine (citicoline) treatment on retinal function and neural conduction in the visual pathways of glaucoma patients with moderate visual defects. Improvement of retinal function (objectively evaluated by pattern electroretinogram recordings) and of neural conduction along visual pathways (objectively evaluated by visual evoked potential recordings) were observed in glaucoma patients after two 60-day periods of oral or intramuscular treatment with citicoline. However, partial regression of this improvement was detected after two 120-day periods of washout. This suggests that the beneficial effects observed are in part treatment-dependent. The extension of citicoline treatment up to a period of 8 years lead to the stabilization or improvement of the glaucomatous visual dysfunction. These results suggest potential neuroprotective effects of citicoline in the glaucomatous disease.
\end{abstract}

Keywords: citicoline; glaucoma; visual evoked potentials; pattern electroretinogram; innermost retinal layers; visual pathways; visual function

\section{Introduction}

The glaucomatous disease open angle glaucoma (OAG) is commonly characterized by the presence of increased intraocular pressure (IOP), typical optic nerve head cupping, and visual field defects,

\footnotetext{
*Corresponding author. Tel.: + 390685356727;

Fax: + 390686216880; E-mail: vparisi@tin.it
}

in particular, evaluated by Humphrey Field Analyzer (HFA) (Graham et al., 1996).

HFA perimetry, however, does not selectively reveal which structures contribute to the impairment of the visual system observed in glaucoma. Alternatively, electrophysiological methods may allow us to explore the different structures that contribute to visual function.

The function of retinal preganglionic elements can be objectively evaluated by recording electroretinographic signals evoked by flash stimuli (flash 
electroretinogram, ERG) (Armington, 1974). Studies in animals (Maffei and Fiorentini, 1982) and humans (Holder, 1997; Parisi et al., 1999d; Parisi, 2003) suggest that the function of retinal ganglion cells (RGCs) and their fibers can be assessed by electroretinographic signal recordings evoked by pattern stimuli (pattern ERG, PERG). The function of the entire visual pathway can be assessed by recording cortical potentials evoked by patterned stimuli (visual evoked potentials, VEPs) (Celesia et al., 1993).

Several studies performed in groups of patients with ocular hypertension without visual field defects (OHT patients) or in groups of patients with glaucoma showed the presence of normal or impaired flash ERG responses (Gur et al., 1987; Vaegan et al., 1995), impaired PERG (Porciatti et al., 1987; Watanabe et al., 1989; Parisi, 1997, 2001; Parisi et al., 1997, 1999c, 2001, 2006; Salgarello et al., 1999; Bach, 2001; Garway-Heath et al., 2002; Ventura et al., 2005), and VEP (Parisi and Bucci, 1992; Greenstein et al., 1998; Martus et al., 1998; Klistorner and Graham, 1999; Parisi, 1997, 2001; Horn et al., 2002; Parisi et al., 1997, 1999c, 2001, 2006) responses when compared to responses obtained in groups of normal subjects.

The results provided by electrophysiological studies suggest that the natural history of glaucoma involves the early impairment of the innermost retinal layers (which may precede the onset of visual field defects) (Parisi et al., 2006), which may be followed by an impairment, due to transynaptic degeneration, in postretinal visual pathways and in particular at the level of the lateral geniculate nucleus (LGN) (Chaturvedi et al., 1993; Weber et al., 2000; Yucel et al., 2000, 2003; Gupta and Yucel, 2003). These observations lead us to believe that the glaucomatous disease must not be considered as a disease exclusively involving ocular structures, but is a pathology in which visual brain structures are also compromised.

Thus, since 1996, we are paying our attention on the possibility of inducing an improvement of visual function using a pharmacological approach similar to that used in different brain disorders ascribed to vascular, traumatic, or degenerative processes (Boismare et al., 1978; Serra et al., 1981; Agnoli et al., 1985; Zappia et al., 1985; Kakihana et al., 1988; Cacabelos et al., 1996).
In particular, in our two previously published studies, we observed that the glaucomatous visual impairment may be improved by treatment with nicergoline (Parisi et al., 1999a) or with cytidine5'-diphosphocholine (citicoline) (Parisi et al., 1999b). Nevertheless, the beneficial effects of these treatments are treatment-dependent. In particular, 45 and 300 days (respectively for nicergoline and citicoline) (Parisi et al., 1999a, b) after the end of the treatment, no differences were detected with respect to pretreatment conditions. When a second period of citicoline treatment was performed, we observed that even after a long period of washout (120 days), it is possible to once again detect visual function improvement, suggesting that repeated treatments may inhibit the development of the visual impairment (Parisi et al., 1999b).

In the above-mentioned study (Parisi et al., 1999b), citicoline treatment was performed by intramuscular injection. As this administration route requires suitable paramedical abilities, it could represent a real problem for patients' selfadministration and thus patient compliance.

At present, citicoline is available as an oral formulation. This can represent an advantage for many patients whose visual dysfunction, as observed in previously published studies, can improve after citicoline treatment.

This chapter presents data regarding studies that evaluated the effects of oral or intramuscular treatment with citicoline on the function of the retina and visual pathways. In these studies, the effects of citicoline on visual field sensitivity were purposely not considered because any detectable improvement could be ascribed to the associated effects on consciousness level and attention (Zappia et al., 1985).

In addition, this chapter discusses whether citicoline effects may be considered as "neuroprotective", which is able to prevent the development of the glaucomatous disease.

\section{Patients: selection and recruitment criteria}

Seventy eyes of 60 patients (range: 38-62 years, mean age $52.77 \pm 5.28$ years) affected by $\mathrm{OAG}$ 
with moderate visual field defects [HFA with mean deviation (MD) between -2 and $-14 \mathrm{~dB}$; corrected pattern standard deviation (CPSD) between +2 and $+12 \mathrm{~dB}$ ] were studied. OAG patients were selected from a very large population (172 OAG patients) on the basis of the inclusion criteria extensively reported in our previously published study (Parisi et al., 2006).

Because it is known that PERG responses can be modified by the pharmacological reduction of IOP (Colotto et al., 1995; Ventura and Porciatti, 2005), we only enrolled OHT and OAG patients with IOP values less than $18 \mathrm{mmHg}$ on beta-blocker monotherapy maintained during the 8 months preceding the electrophysiological evaluation.

All OAG patients were randomly divided on the basis of age and visual field defects into three groups (see below):

- Group NT-OAG (not treated open-angle glaucoma): 20 OAG patients (range: 39-61 years, mean age $51.64 \pm 4.68$ years, 20 eyes) in which no additional pharmacological treatment was performed.

- Group TI-OAG (glaucoma patients treated with intramuscular citicoline): 20 OAG patients (range: 41-62 years, mean age $52.54 \pm 5.43$ years, 20 eyes) treated with a daily intramuscular dose of $1000 \mathrm{mg}$ citicoline (Cebroton $1000^{\circledR}$, Tubilux Pharma, Pomezia, Rome, Italy).

- Group TO-OAG (glaucoma patients treated with oral citicoline): $20 \mathrm{OAG}$ patients (range: $38-60$ years, mean age $50.84 \pm 6.62$ years, 20 eyes) treated with a daily oral dose of $1600 \mathrm{mg}$ citicoline (Cebrolux ${ }^{\circledR}$, Tubilux Pharma).

The research followed the tenets of the Declaration of Helsinki. The protocol was approved by the local Institutional Review Board (IRB). Upon recruitment, each patient gave informed consent.

\section{Pharmacological treatment protocol}

The pharmacological treatment was performed according to the following schedule.

First period - A daily intramuscular dose of $1000 \mathrm{mg}$ citicoline or oral dose of $1600 \mathrm{mg}$ citicoline was prescribed according to the following protocol:

- 0-60 days: First period of pharmacological treatment with oral or intramuscular citicoline.

- 61-180 days: First period of washout and follow-up at the sixth month.

Second period - A daily intramuscular dose of $1000 \mathrm{mg}$ citicoline or oral dose of $1600 \mathrm{mg}$ citicoline was prescribed according to the following protocol:

- 181-240 days: Second period of pharmacological treatment with oral or intramuscular citicoline.

- 241-360 days: Second period of washout and follow-up at the twelfth month.

\section{Methodology of visual function evaluation: electrophysiological examinations}

PERG and VEP recordings were performed using the following methods (Parisi, 1997, 2001; Parisi et al., 1997, 1999c, 2001, 2006).

Briefly, subjects were seated in a semidark, acoustically isolated room in front of the display surrounded by a uniform field of luminance of $5 \mathrm{~cd} / \mathrm{m}^{2}$. Prior to the experiment, each subject was adapted to the ambient room light for $10 \mathrm{~min}$ and pupil diameter was approximately $5 \mathrm{~mm}$. Mydriatic or miotic drugs were never used. Stimulation was monocular after occlusion of the other eye. Visual stimuli were checkerboard patterns (contrast $80 \%$, mean luminance $110 \mathrm{~cd} / \mathrm{m}^{2}$ ) generated on a TV monitor and reversed in contrast at the rate of 2 reversals per second; at the viewing distance of $114 \mathrm{~cm}$, the check edges subtended 15 minutes $\left(15^{\prime}\right)$ of visual angle. The monitor screen subtended $18^{\circ}$. PERG and VEP recordings were performed with full correction of refraction at the viewing distance. A small red fixation target, subtending a visual angle of approximately 0.5 degrees (estimated after taking into account spectacle-corrected individual refractive errors) was placed at the center of the pattern stimulus. At every PERG and VEP examination, each 
patient positively reported that he/she could clearly perceive the fixation target. The refraction of all subjects was corrected for viewing distance.

\section{PERG recordings}

The bioelectrical signal was recorded by a small $\mathrm{Ag} / \mathrm{AgCl}$ skin electrode placed over the lower eyelid. PERGs were derived bipolarly between the stimulated (active electrode) and the patched (reference electrode) eye using a previously described method (Fiorentini et al., 1981). As the recording protocol was extensive, the use of skin electrodes with interocular recording represented a good compromise between signal-to-noise ratio (SNR) and signal stability. The ground electrode was in Fpz. Interelectrode resistance was lower than 3000 ohms. The signal was amplified (gain 50,000 ), filtered (band pass $1-30 \mathrm{~Hz}$ ), and averaged with automatic rejection of artifacts (200 events free from artifacts were averaged for every trial) by BM 6000 (Biomedica Mangoni, Pisa, Italy). Analysis time was $250 \mathrm{~ms}$. The transient PERG response is characterized by a number of waves with three subsequent peaks, of negative, positive, negative polarity, respectively. In normal subjects, these peaks have the following implicit times: 35 , 50, and $95 \mathrm{~ms}$ (N35, P50, N95).

\section{VEP recordings}

Cup-shaped electrodes of $\mathrm{Ag} / \mathrm{AgCl}$ were fixed with collodion in the following positions: active electrode in $\mathrm{Oz}$, reference electrode in Fpz, and ground in the left arm. Interelectrode resistance was kept below $3000 \mathrm{ohms}$. The bioelectric signal was amplified (gain 20,000), filtered (band pass $1-100 \mathrm{~Hz}$ ), and averaged (200 events free from artifacts were averaged for every trial) by BM 6000. Analysis time was $250 \mathrm{~ms}$. The transient VEP response is characterized by a number of waves with three subsequent peaks, of negative, positive, negative polarity, respectively. In normal subjects, these peaks have the following implicit times: 75, 100, and $145 \mathrm{~ms}$ (N75, P100, N145).

During a recording session, simultaneous VEPs and PERGs were recorded at least twice (2-6 times) and the resulting waveforms were superimposed to check the repeatability of the results. All control, OHT, and OAG eyes underwent at least two recording sessions, 1-7 days apart, to determine test-retest variability.

In each subject or patient, the SNR of PERG and VEP responses was assessed by measuring a "noise" response, while the subject fixated at an unmodulated field of the same mean luminance as the stimulus. At least two "noise" records of 200 events each were obtained and the resulting grand average was considered for measurement. The peak-to-peak amplitude of this final waveform (i.e., average of at least two replications) was measured in a temporal window corresponding to that at which the response component of interest (i.e., VEP N75-P100, PERG P50-N95) was expected to peak. SNRs for this component were determined by dividing the peak amplitude of the component by the noise in the corresponding temporal window. An electroretinographic noise $<0.1 \mu \mathrm{V}$ (mean: $0.085 \mu \mathrm{V}$, range: $0.065-0.095 \mu \mathrm{V}$, resulting from the grand average of $400-1200$ events) and an evoked potential noise $<0.15 \mu \mathrm{V}$ (mean: $0.093 \mu \mathrm{V}$, range: $0.072-0.112 \mu \mathrm{V}$, resulting from the grand average of 400-1200 events) were observed in all tested subjects. In all subjects and patients, we accepted VEP and PERG signals with an SNR > 2 .

Implicit time and peak amplitude of each wave were measured directly on the displayed records by means of a pair of cursors for all VEPs and PERGs. Simultaneous recordings of VEPs and PERGs allow us to derive Retinocortical Time (RCT) as the difference between VEP P100 and PERG P50 peak latencies (Celesia and Kaufmann, 1985).

At baseline, all OAG patients underwent at least two simultaneous recordings of PERG and VEP 1-7 days apart to determine test-retest variability. During the follow-up assessment $(60,180,240$, and 360 days), PERGs and VEPs were performed in $\mathrm{OAG}$ patients at least three times and the resulting waveforms were superimposed to check the repeatability of the results. The recording with the highest PERG P50-N95 amplitude was considered in the statistical analysis (see below). During all follow-up examinations, PERG and VEP recordings were performed in a condition of pupil 
diameter equal to that measured in baseline conditions (see above).

\section{Statistic evaluation of electrophysiological results}

Sample size estimates were obtained from pilot evaluations performed in 20 eyes from 20 OAG patients and 20 eyes from 20 control subjects, other than those included in the current study. Interindividual variability, expressed as data standard deviation (SD), was estimated for PERG P50-N95 amplitude and VEP P100 implicit time measurements. It was found that data SDs were significantly higher for patients when compared to controls (about $35 \%$ vs. 15\%). It was also established that, assuming the above betweensubjects SD in the current study, sample sizes of control subjects and patients belonging to OAG group provided a power of $90 \%$, at an alpha $=0.05$, for detecting a between-group difference of $55 \%$ or greater in PERG P50-N95 amplitude and VEP P100 implicit time measurements. These differences were preliminarily observed by comparing OAG and control data (see above). They were also expected to be clinically meaningful when comparing results of treated OAG eyes observed in baseline conditions versus those observed at $60,180,240$, and 360 days.

Test-retest data of PERG and VEP results were expressed as the mean difference between two recordings obtained in separate sessions plus/ minus the SD of this difference. Ninety-five percent confidence limits of test-retest variability in normal subjects and patients were established assuming a normal distribution.

The differences of PERG and VEP responses between groups (NT-OAG, TO-OAG, and TI-OAG eyes) were evaluated by one-way analysis of variance (ANOVA). Changes in PERG and VEP responses observed in NT-OAG, TO-OAG, and TIOAG groups with respect to baseline were evaluated by one-way repeated measures ANOVA. The differences observed in individual TO-OAG eyes after observation or citicoline treatment with respect to baseline values were calculated by performing a logarithmic transformation to better approximate a normal distribution. In all analyses, a conservative $p$ value less than 0.01 , to compensate for multiple comparisons, was considered as statistically significant.

\section{Electrophysiological (PERG and VEP) responses in OAG patients after the first period of evaluation}

Examples of simultaneous recordings of VEP and PERG before and after treatment with oral citicoline are displayed in Fig. 1. Individual changes observed in TO-OAG eyes are shown in Fig. 2. Mean values observed in NT-OAG, TI-OAG, and TO-OAG eyes are presented in Figs. 3 and 4.

A decrease in PERG P50 implicit time, VEP P100 implicit time, and RCT, and an increase in PERG P50-N95 and VEP N75-P100 amplitudes $(p<0.01)$ were found in TO-OAG and TI-OAG patients after 60 days of treatment (day 60) with respect to baseline values. TO-OAG and TI-OAG patients displayed shorter PERG P50 implicit time, VEP P100 implicit time, and RCT, and greater PERG and VEP amplitudes with respect to NT-OAG patients $(p<0.01)$.

Increased PERG P50 implicit time, VEP P100 implicit time, and RCT, and decreased PERG P50N95 and VEP N75-P100 amplitudes were found at day 180 with respect to values observed at 60 days. PERG and VEP parameters observed were still shorter (P50 implicit time, P100 implicit time, RCT) and still greater (P50-N95 and N75-P100 amplitudes) with respect to those observed in baseline conditions $(p<0.01)$ and with respect to those observed in NT-OAG patients $(p<0.01)$.

No changes $(p>0.01)$ in PERG and VEP responses were observed in NT-OAG patients after 60 and 180 days with respect to baseline conditions.

\section{Electrophysiological (PERG and VEP) responses in} OAG patients after the second period of evaluation

A further decrease in PERG P50 implicit time, VEP P100 implicit time, and RCT, and a further increase in PERG P50-N95 and VEP N75-P100 amplitudes $(p<0.01)$ were observed in TO-OAG 

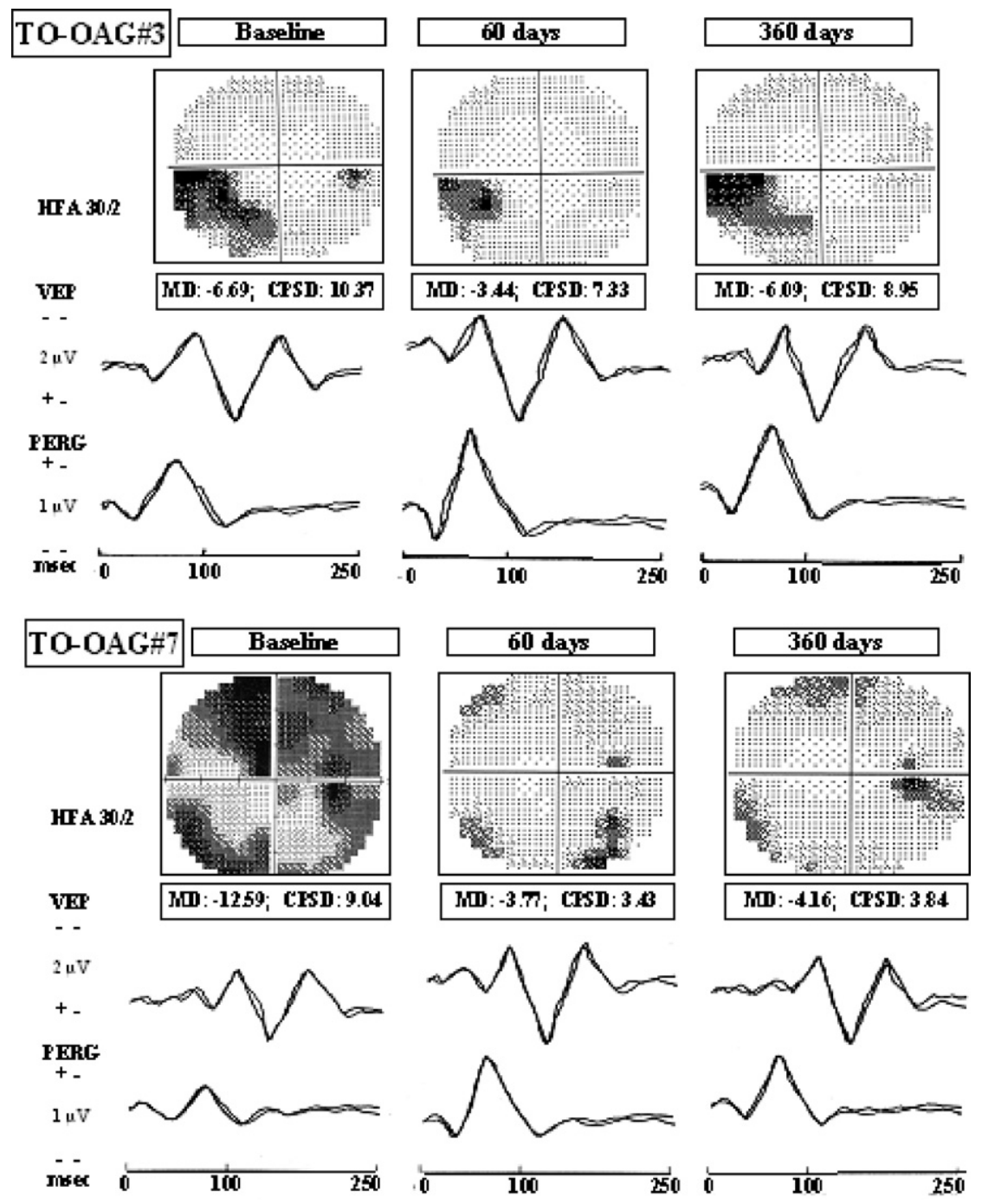

Fig. 1. Examples of visual field (Humphrey 30-2) and layouts of simultaneous visual evoked potentials (VEPs) and pattern electroretinograms (PERGs) recorded in two patients affected by open-angle glaucoma treated with oral citicoline (TO-OAG\#3, TO-OAG\#7). Electrophysiological examinations were assessed at baseline conditions and 60, 180, 240, and 360 days after medical treatment with oral citicoline. Oral citicoline treatment was performed in two different 60-day periods (0-60 and 181-240 days), each followed by a period of washout (61-180 and 241-360 days). TO-OAG patients showed a decrease in implicit times and an increase in amplitude after the first period of citicoline treatment (60 days) when compared to baseline conditions. At the end of the second period of washout (360 days), one OAG patient (TO-OAG\#3) showed electrophysiological and visual field parameters similar to those observed in baseline conditions, while in the other example (TO-OAG\#7), the visual field and electrophysiological improvements observed after oral citicoline treatment remained stable even after the washout period.

and TI-OAG patients, at day 240 , with respect to values observed at day 180. PERG and VEP implicit times and RCT were still shorter and PERG and VEP amplitudes were still greater when compared to NT-OAG patients $(p<0.01)$. At day
360, TO-OAG and TI-OAG patients showed an increase in VEP and PERG implicit times and in $\mathrm{RCT}$, and a decrease in amplitudes with respect to values observed at 240 days. Implicit time and amplitude values were respectively still shorter and 

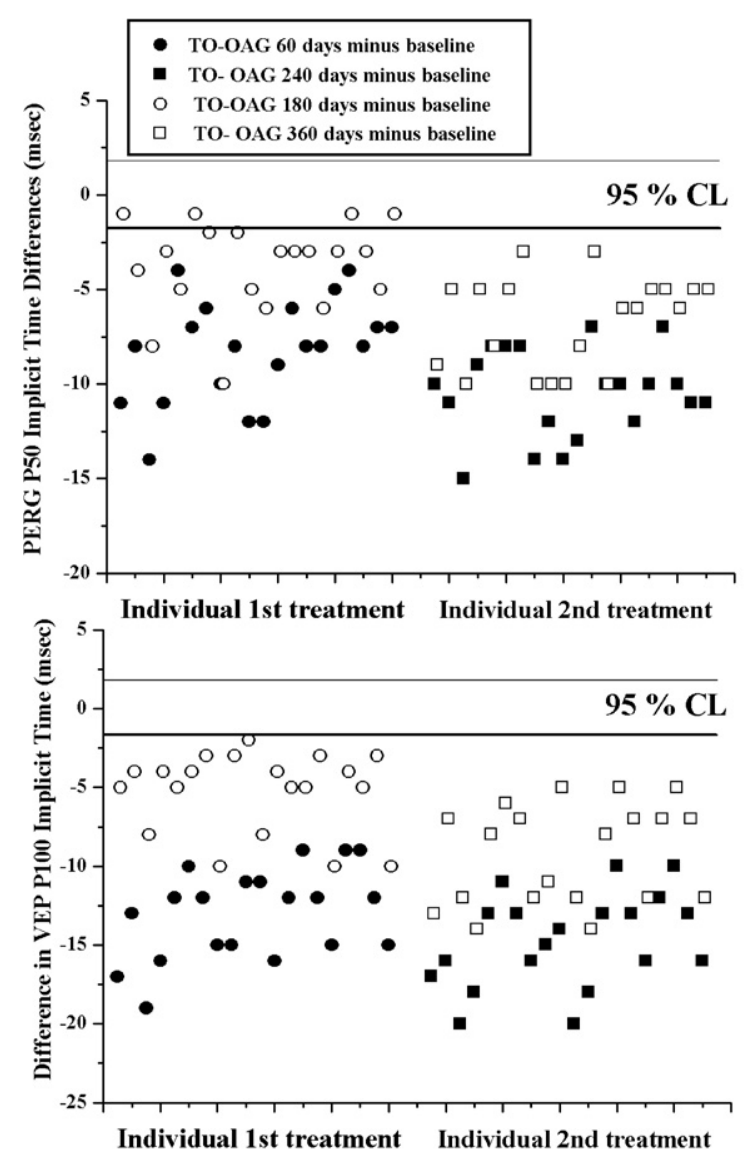
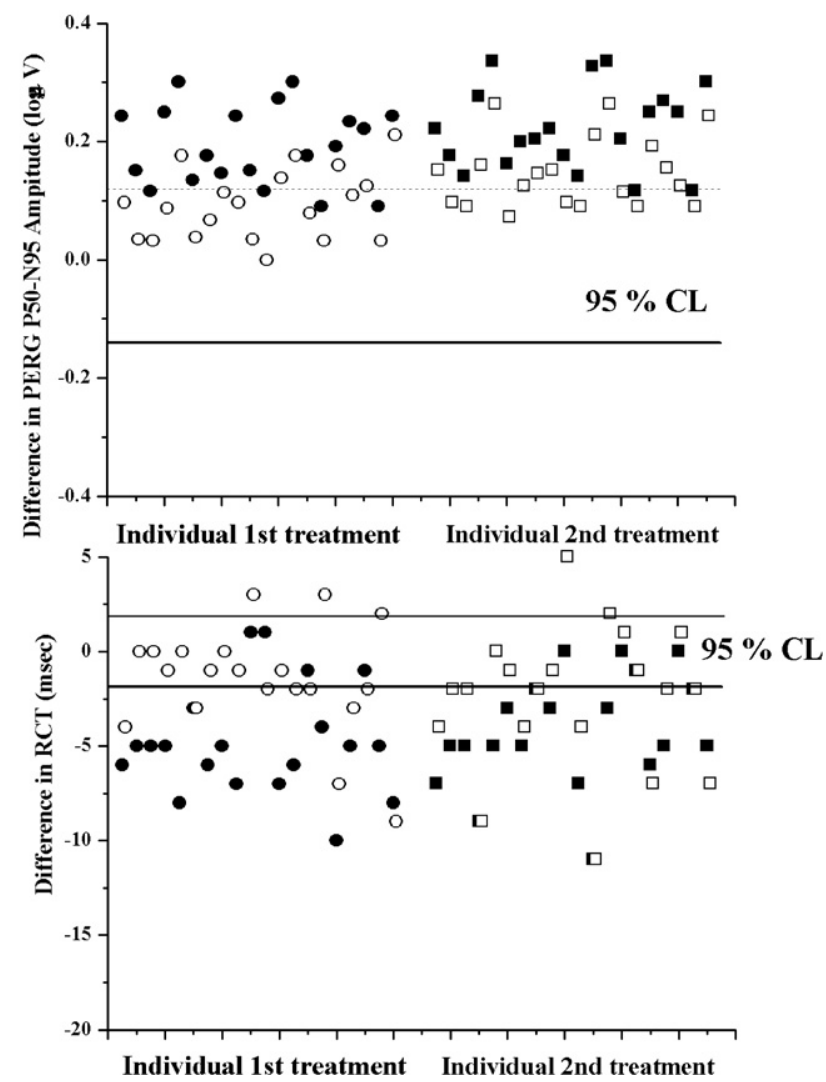

Fig. 2. Individual changes in pattern electroretinogram (PERG), visual evoked potential (VEP) responses, and retinocortical time (difference between VEP P100 and PERG P50 implicit times and RCT) observed in eyes affected by open-angle glaucoma treated with oral citicoline (TO-OAG). Values refer to the difference observed after 60, 180, 240, and 360 days with respect to baseline conditions. Medical treatment with citicoline was performed in TO-OAG eyes over a 60-day period followed by 120 days of washout. At day 180 , a second 60-day period of citicoline treatment followed by a second period of 180 days of washout was performed. Solid and dashed lines refer respectively to the upper and lower $95 \%$ confidence limit of the intraindividual variability resulting from test-retest analysis.

still greater when compared to baselines $(p<0.01)$ and when compared to NT-OAG patients $(p<0.01)$.

No changes $(p>0.01)$ in PERG and VEP responses were observed in NT-OAG patients, after 240 and 360 days, with respect to baseline conditions.

Considering electrophysiological responses observed after the two different periods of treatment or washout, no significant differences $(p>0.05)$ were found between TO-OAG and TI-OAG patients when comparing VEP and PERG changes in implicit times or amplitudes (see Fig. 4).
Adverse side effects were not reported by any of the patients enrolled in the study during the entire period of treatment. Significant changes in IOP were not found in any of the subjects tested.

\section{Effects of citicoline on retinal function in glaucoma patients: neurophysiological implications}

Oral or intramuscular treatment with citicoline induces an improvement of retinal bioelectric responses in our glaucomatous patients, as suggested by the increase in amplitudes and shortening in implicit times of PERG recordings. 

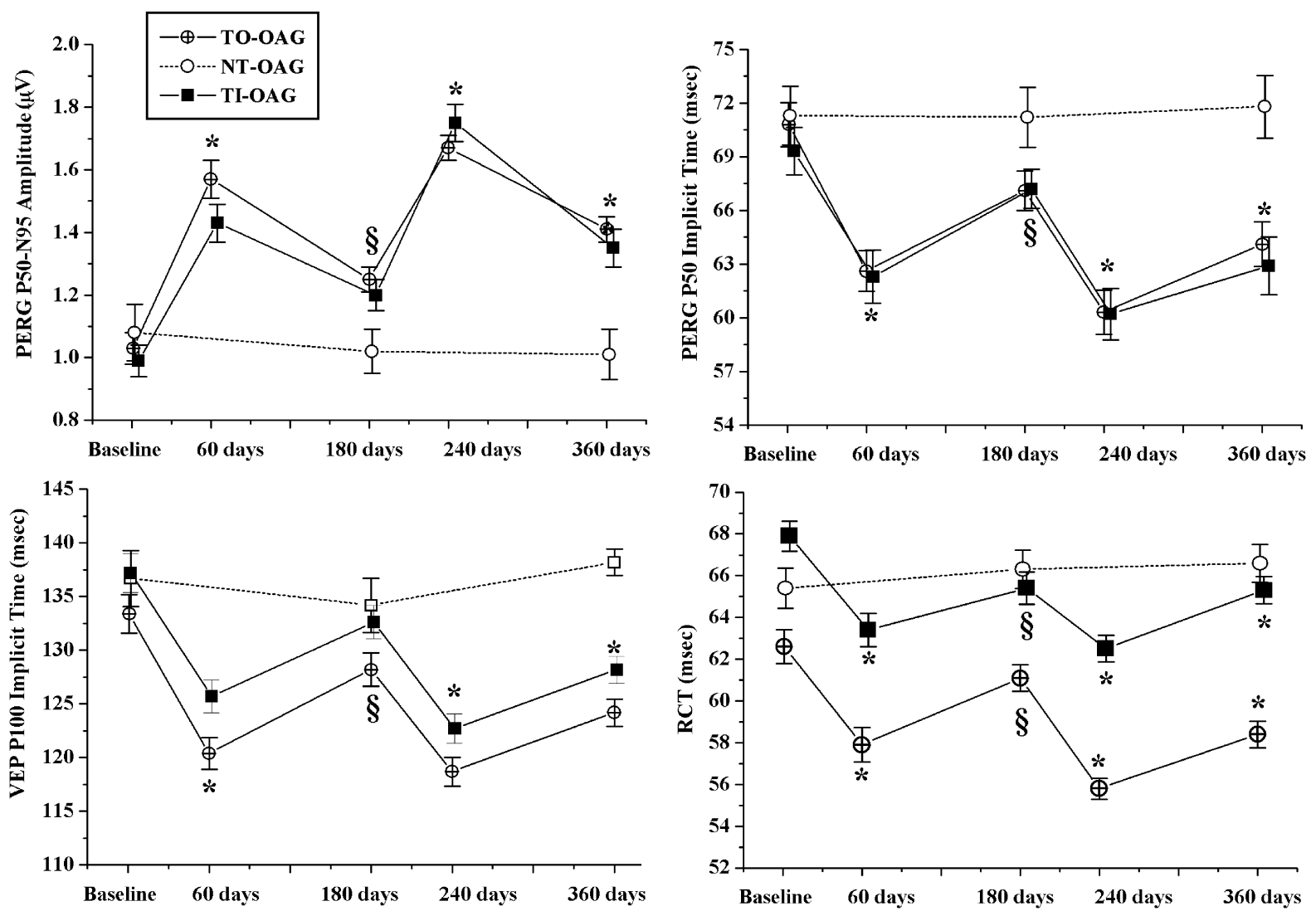

Fig. 3. Graphic representation of mean values of PERG P50 implicit time and P50-N95 amplitude, VEP P100 implicit time, and RCT observed in patients affected by open-angle glaucoma without additional treatments (NT-OAG) and in patients with glaucoma treated with oral (TO-OAG) or intramuscular (TI-OAG) citicoline. Solid lines indicate periods of medical treatment, while dashed lines indicate periods of washout. Vertical lines represent one standard error of the mean. Statistical analysis (ANOVA) evaluating the differences between TO-OAG and TI-OAG groups versus baseline conditions and with respect to NT-OAG group: ${ }^{*} p<0.01 ;{ }^{\S} p>0.01$.

In our studies we found that there were no differences between oral and intramuscular treatment with citicoline. The results obtained with intramuscular treatment are consistent with those observed in our previous study (Parisi et al., 1999b).

Although in this study, just as in our previous study (Parisi et al., 1999b), our results clearly suggest a positive effect of citicoline in improving the retinal and postretinal glaucomatous dysfunction, the mechanism of action of citicoline in the visual system is only in part understood.

Citicoline is an endogenous substance that acts as intermediary in the synthesis of phosphatidylcholine (a major phospholipid in the neuronal membrane) (Kennedy, 1957; Goracci et al., 1985; Secades and Frontera, 1995; Weiss, 1995) through the activation of the biosynthesis of structural phospholipids in neuronal membranes. Citicoline increases the metabolism of cerebral structures (Secades and Frontera, 1995) and inhibits phospholipid degradation (Weiss, 1995). It may therefore have potential neuroprotective and neuromodulator roles as demonstrated in conditions of cerebral hypoxia and ischemia (Secades and Frontera, 1995; Weiss, 1995) and by the evidence that it induces an increase in the levels of different neurotransmitters and neuromodulators, including noradrenaline, in the central nervous 

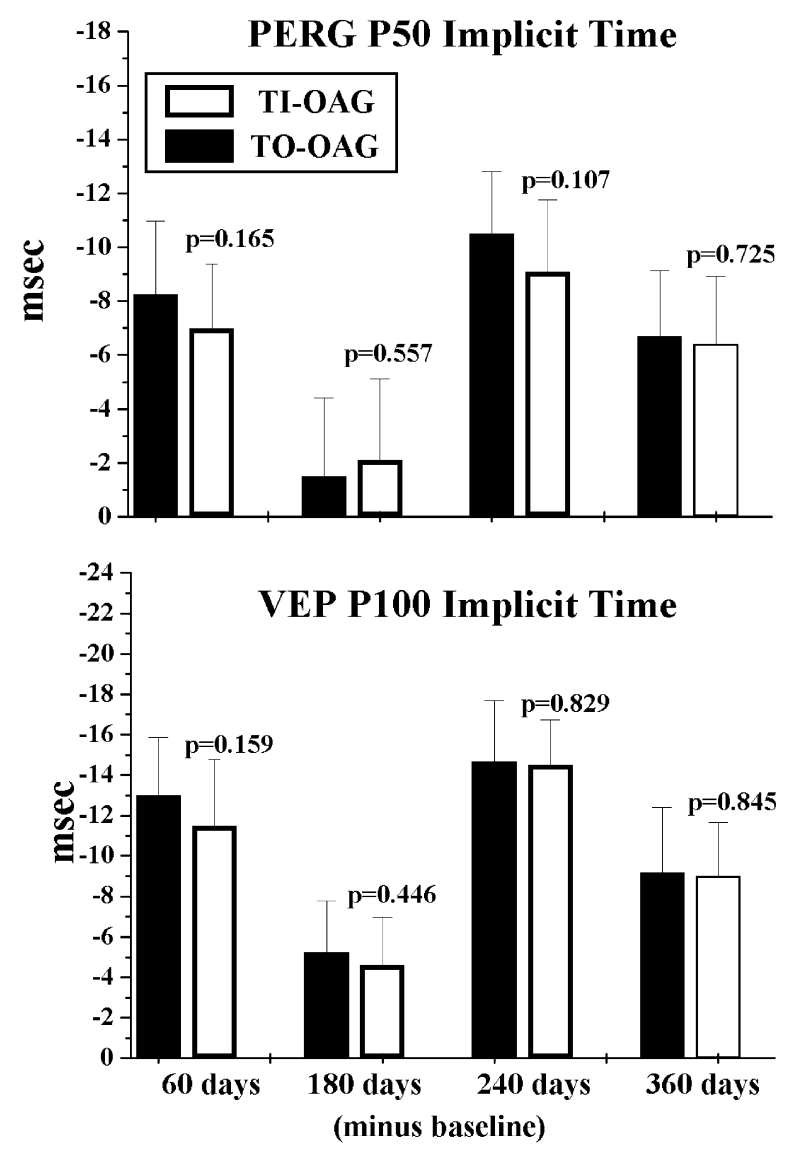
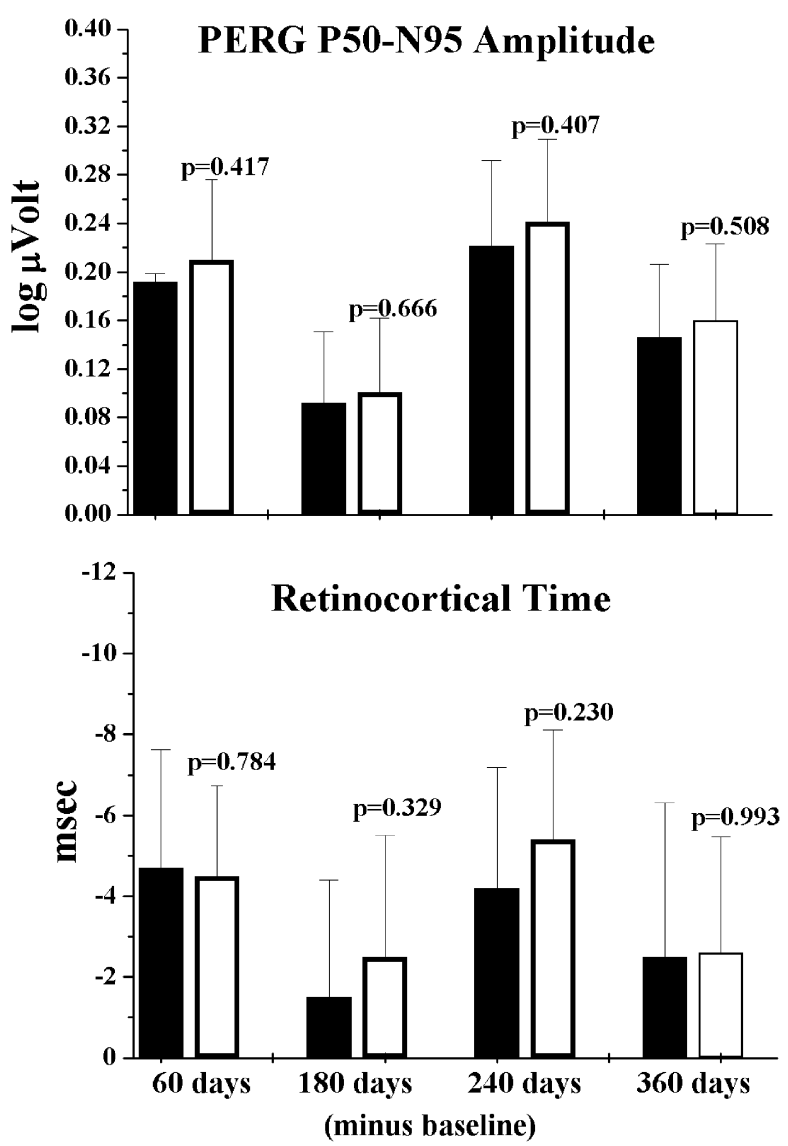

Fig. 4. Bar graphs of the mean differences observed after oral or intramuscular citicoline treatment (60 and 240 days) or after washout (180 and 360 days) with respect to baseline conditions. Means refer to changes in PERG P50 implicit time and P50-N95 amplitude, VEP P100 implicit time, and RCT observed in patients affected by open-angle glaucoma treated with oral (TO-OAG) or intramuscular (TI-OAG) citicoline. Vertical lines represent one standard deviation of the mean. The $p$ value refers to a statistical analysis (ANOVA) evaluating differences between TI-OAG and TO-OAG groups.

system. In addition, several studies suggest that citicoline successfully increases the level of consciousness in different brain disorders ascribed to vascular, traumatic, or degenerative processes (Boismare et al., 1978; Serra et al., 1981; Agnoli et al., 1985; Zappia et al., 1985; Kakihana et al., 1988; Cacabelos et al., 1996).

Previous studies report that treatment with citicoline may induce an improvement of the glaucomatous visual field defects (Pecori-Giraldi et al., 1989; Virno et al., 2000). In our study, the effects of citicoline on visual field sensitivity were purposely not considered because any detectable improvement could be ascribed to the associated effects on consciousness level and attention (Boismare et al., 1978; Serra et al., 1981; Agnoli et al., 1985; Zappia et al., 1985; Kakihana et al., 1988; Cacabelos et al., 1996).

A dopaminergic-like activity of citicoline may also be involved in the improvement of PERG responses after oral treatment; in fact, levodopa was found to increase retinal function in humans treated with this substance (Gottlob et al., 1989), and our results could therefore be explained by a similar neuromodulator activity.

In our study, we did not perform any morphological examination, and thus, although our results indicate that oral citicoline improves bioelectrical 
retinal activity, we were not able to demonstrate whether there were other effects on retinal fibers (i.e., an increase in retinal nerve fiber layer thickness).

\section{Effects of citicoline on neural conduction along the visual pathways in glaucoma patients: neurophysiological implications}

Oral or intramuscular treatment with citicoline induced an improvement of visual cortical bioelectric responses (VEPs with increased amplitudes and shortened times to peak) and an improvement of the index of neural conduction in postretinal visual pathways (reduced RCT) in our glaucomatous patients. This is in agreement with a similar previous study (Rejdak et al., 2003).

VEP abnormalities observed in patients with glaucoma have recently been ascribed to impaired neural conduction along postretinal visual pathways related to a dysfunction of the innermost retinal layers (ganglion cells and their fibers) (Parisi, 2001). An independent effect on neural conduction in postretinal visual pathways or in visual cortical cells could also be hypothesized in order to explain the influence of citicoline in VEP responses.

The effects of citicoline in the neural visual system were revealed by the improvement of visual acuity (Campos et al., 1995; Porciatti et al., 1998), VEP responses, and contrast sensitivity (Porciatti et al., 1998) in amblyopic subjects after treatment with this substance. Because similar results were obtained in amblyopic subjects after treatment with levodopa (Gottlob and Stangler-Zuschrott, 1990; Gottlob et al., 1992; Leguire et al., 1993) and studies performed in patients with Parkinson's disease recommended the use of citicoline as a complement to levodopa therapy (Birbamer et al., 1990), a dopaminergic-like activity could once again be suggested to explain VEP and RCT results after treatment with citicoline.

Because there are no clear or conclusive experimental or published data to support the hypothesis of an independent effect on neural conduction in postretinal visual pathways, the changes in neural conduction along the visual pathways following citicoline treatment could be dependent on the reduced dysfunction of the innermost retinal layers.

\section{Possibility of neuroprotective role of citicoline in glaucoma patients}

Our results advocate an interesting question: can oral or intramuscular citicoline effects be considered as "neuroprotective," preventing the development of the glaucomatous disease?

For citicoline effects to be considered "neuroprotective," glaucomatous visual defects should undergo stabilization or improvement long after treatment termination.

When considering that after the first period of washout there were no differences with respect to pretreatment conditions, this leads to the conclusion that one cycle of treatment with citicoline is not sufficient to induce changes in the natural history of glaucoma. On the other hand, we observed that the second treatment period with oral citicoline was able to induce an improvement, which persisted after 120 days of washout.

These observations suggest that the improvement in retinal function and postretinal neural conduction along the visual pathways could be considered as "treatment-dependent."

The results obtained in our first study (Parisi et al., 1999b) suggested us to perform in a restrict cohort of selected patients (12 OAG patients only) a series of 60-day periods of treatment each followed by 120 days of washout during a total period of 8 years (Parisi, 2005). This study showed that after 8 years, glaucomatous patients subjected to citicoline treatment displayed a stable or improved electrophysiological and visual field impairment with respect to pretreatment conditions (8 years before), while in similar glaucomatous patients not subjected to citicoline treatment, there was a worsening of the electrophysiological and visual field impairment with respect to pretreatment conditions ( 8 years before). The results of this study are summarized in Fig. 5.

Indeed, the data observed in our OAG patients treated with beta-blockers plus several periods of treatment with intramuscular citicoline with respect to those found in OAG patients treated with beta-blockers only may suggest the potential use of citicoline in order to obtain the stabilization or improvement of the glaucomatous visual dysfunction. 

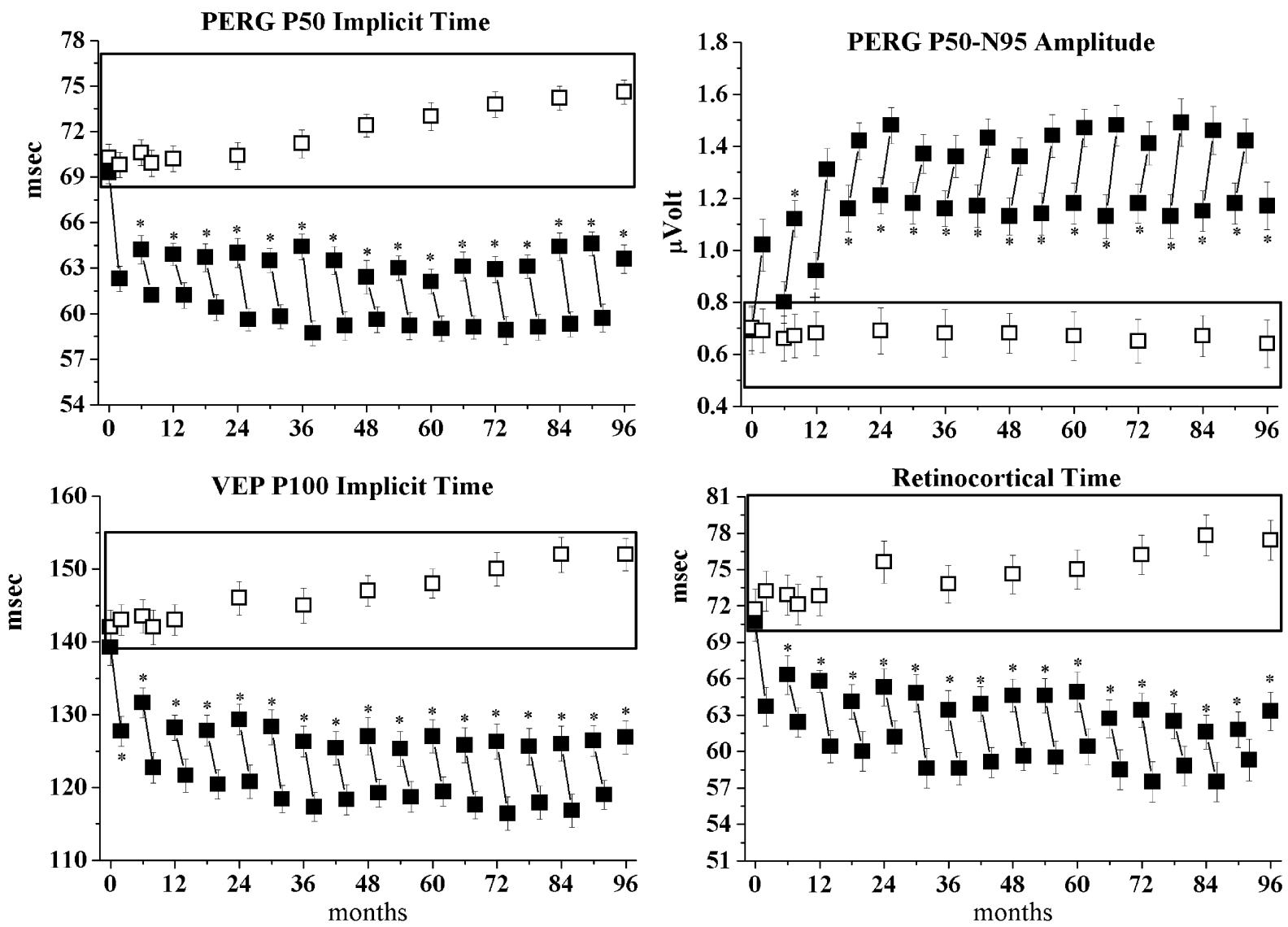

Fig. 5. Graphic representation of mean values of PERG P50 implicit time, PERG P50-N95 amplitude, VEP P100 implicit time, and retinocortical time (difference between VEP P100 and PERG P50 implicit times, an electrophysiological index of neural conduction along postretinal visual pathways) observed in glaucoma patients at baseline conditions and without additional treatment (hollow bar) or treated with intramuscular citicoline (solid bar). Medical treatment with citicoline was performed over several 2-month periods followed by several 4-month periods of washout during an entire period of 96 months. The solid line within solid bars indicates periods of treatment. The absence of a line within a solid bar indicates washout periods. Vertical lines represent one standard error of the mean. We concentrated on the long-term effects of citicoline treatment by comparing the differences observed at the end of each period of washout with respect to baseline conditions through ANOVA. ${ }^{*} p<0.01$ versus baseline and untreated glaucoma patients. Adapted with permission from Parisi, 2005 (Figs. 2-5, pp. 98-99).

These observations suggest that citicoline could be considered a neuroprotective drug. Our study showed that the neuroprotective effects of citicoline may be achieved in a restricted cohort of patients (see inclusion criteria, Parisi et al., 2006) and must be considered "treatment-dependent," leading to the possibility that repeated treatments may determine possible neuroprotective effects.

In agreement with the reported observations of similar studies (Kennedy, 1957; Campos et al., 1995; Secades and Frontera, 1995; Weiss, 1995; Porciatti et al., 1998; Parisi et al., 1999b; Parisi, 2005), an important aspect of this study is the lack of adverse pharmacological side effects in all participating subjects, even after long-term administration of the drug.

\section{Conclusive remarks}

The results provided by our studies suggest that oral and intramuscular citicoline treatment 
significantly improves retinal and cortical responses in glaucoma patients. This indicates the potential use of this substance in the medical treatment of glaucoma, as a complement to hypotensive therapy, with a possible direct neuroprotective effect.

\begin{tabular}{|c|c|}
\hline \multicolumn{2}{|c|}{ Abbreviations } \\
\hline CPSD & $\begin{array}{l}\text { Corrected Pattern Standard } \\
\text { Deviation }\end{array}$ \\
\hline ERG & electroretinogram \\
\hline HFA & Humphrey Field Analyzer \\
\hline IOP & intraocular pressure \\
\hline IRB & Institutional Review Board \\
\hline LGN & lateral geniculate nucleus \\
\hline MD & mean deviation \\
\hline NT-OAG & not treated open-angle glaucoma \\
\hline OAG & open-angle glaucoma \\
\hline OHT & ocular hypertension \\
\hline PERG & pattern electroretinogram \\
\hline RCGs & retinal ganglion cells \\
\hline $\mathrm{RCT}$ & retinocortical time \\
\hline SD & standard deviation \\
\hline SNR & signal-to-noise ratio \\
\hline TI-OAG & $\begin{array}{l}\text { glaucoma patients treated with } \\
\text { intramuscular citicoline }\end{array}$ \\
\hline TO-OAG & $\begin{array}{l}\text { glaucoma patients treated with } \\
\text { oral citicoline }\end{array}$ \\
\hline VEPs & visual evoked potentials \\
\hline
\end{tabular}

\section{References}

Agnoli, A., Fioravanti, M. and Lechner, H. (1985) Efficacy of CDP-choline in chronic cerebral vascular diseases (CCVD). In: Novel Biochemical, Pharmacological and Clinical Aspects of Cytidinediphosphocholine. Proceedings of the International Meeting, Sorrento, Italy, 1984. New York: Elsevier, pp. 305308 .

Armington, J.C. (1974) The Electroretinogram. Academic Press, New York.

Bach, M. (2001) Electrophysiological approaches for early detection of glaucoma. Eur. J. Ophthalmol., 11(Suppl 2): S41-S49.

Birbamer, G., Gesterbrand, E., Rainer, J. and Eberhardt, R. (1990) CDP-choline in the treatment of Parkinson's disease. New Trends Clin. Pharmacol., 4: 1-6.

Boismare, F., Le Poncin, M., Lefrancois, J. and Lecordier, J.C. (1978) Action of cytidine diphosphocholine on functional and hemodynamic effects of cerebral ischemia in cats. Pharmacology, 17: 15-20.

Cacabelos, R., Caamano, J., Gomez, M.J., Fernandez-Novoa, L., Franco-Maside, A. and Alvarez, X.A. (1996) Therapeutic effects of CDP-choline in Alzheimer's disease. Cognition, brain mapping, cerebrovascular hemodynamics, and immune factors. Ann. N. Y. Acad. Sci., 777: 399-403.

Campos, E.C., Schiavi, C., Benedetti, P., Bolzani, R. and Porciatti, V. (1995) Effect of citicoline on visual acuity in amblyopia: preliminary results. Graefes. Arch. Clin. Exp. Ophthalmol., 233: 307-312.

Celesia, G.C. and Kaufmann, D. (1985) Pattern ERG and visual evoked potentials in maculopathies and optic nerve disease. Invest. Ophthalmol. Vis. Sci., 26: 726-735.

Celesia, G.G., Bodis-Wollner, I., Chatrian, G.E., Harding, G.F., Sokol, S. and Spekreijse, H. (1993) Recommended standards for electroretinograms and visual evoked potentials. Report of an IFCN committee. Electroencephalogr. Clin. Neurophysiol., 87: 421-436.

Chaturvedi, N., Hedley-Whyte, E.T. and Dreyer, E.B. (1993) Lateral geniculate nucleus in glaucoma. Am. J. Ophthalmol., 116: $182-188$.

Colotto, A., Salgarello, T., Giudiceandrea, A., De Luca, L.A., Coppè, A., Buzzonetti, L. and Falsini, B. (1995) Pattern electroretinogram in treated ocular hypertension: a crosssectional study after timolol maleate therapy. Ophthalmic Res., 27: 168-177.

Fiorentini, A., Maffei, L., Pirchio, M., Spinelli, D. and Porciatti, V. (1981) The ERG in response to alternating gratings in patients with diseases of the peripheral visual pathway. Invest. Ophthalmol. Vis. Sci., 21: 490-493.

Garway-Heath, D.F., Holder, G.E., Fitzke, F.W. and Hitchings, R.A. (2002) Relationship between electrophysiological, psychophysical, and anatomical measurements in glaucoma. Invest. Ophthalmol. Vis. Sci., 43: 2213-2220.

Goracci, G., Francescangeli, E., Mozzi, R., Porcellati, S. and Porcellati, G. (1985) Regulation of phospholipid metabolism by nucleotides in brain and transport of CDP-choline into brain. In: Zappia V., Kennedy E.P., Nilsson B.I. and Galletti P. (Eds.), Novel Biochemical, Pharmacological and Clinical Aspects of Cytidinediphosphocholine. Elsevier, New York, pp. 105-109.

Gottlob, I., Charlier, J. and Reinecke, R.D. (1992) Visual acuities and scotomas after one week levodopa administration in human amblyopia. Invest. Ophthalmol. Vis. Sci., 33: 2722-2728.

Gottlob, I. and Stangler-Zuschrott, E. (1990) Effect of levodopa on contrast sensitivity and scotomas in human amblyopia. Invest. Ophthalmol. Vis. Sci., 31: 776-780.

Gottlob, I., Weghaupt, H., Vass, C. and Auff, E. (1989) Effect of levodopa on the human pattern electroretinogram and pattern visual evoked potentials. Graefes. Arch. Clin. Exp. Ophthalmol., 227: 421-427.

Graham, S.L., Drance, S.M., Chauhan, B.C., Swindale, N.V., Hnik, P., Mikelberg, F.S. and Douglas, G.R. (1996) Comparison of psychophysical and electrophysiological testing in early glaucoma. Invest. Ophthalmol. Vis. Sci., 37: 2651-2662. 
Greenstein, V.C., Seliger, S., Zemon, V. and Ritch, R. (1998) Visual evoked potential assessment of the effects of glaucoma on visual subsystems. Vision Res., 38: 1901-1911.

Gupta, N. and Yucel, Y.H. (2003) Brain changes in glaucoma. Eur. J. Ophthalmol., 13(Suppl 3): S32-S35.

Gur, M., Zeevi, Y.Y., Bielik, M. and Neumann, E. (1987) Changes in the oscillatory potentials of the electroretinogram in glaucoma. Curr. Eye Res., 6: 457-466.

Holder, G.E. (1997) The pattern electroretinogram in anterior visual pathway dysfunction and its relationship to the pattern visual evoked potential: a personal clinical review of 743 eyes. Eye, 11: 924-934.

Horn, F.K., Jonas, J.B., Budde, W.M., Junemann, A.M., Mardin, C.Y. and Korth, M. (2002) Monitoring glaucoma progression with visual evoked potentials of the bluesensitive pathway. Invest. Ophthalmol. Vis. Sci., 43: 1828-1834.

Kakihana, M., Fukuda, N., Suno, M. and Nagaoka, A. (1988) Effects of CDP-choline on neurologic deficits and cerebral glucose metabolism in a rat model of cerebral ischemia. Stroke, 19: 217-222.

Kennedy, E.P. (1957) Biosynthesis of phospholipids. Fed. Proc., 16: 847-853.

Klistorner, A.I. and Graham, S.L. (1999) Early magnocellular loss in glaucoma demonstrated using the pseudorandomly stimulated flash visual evoked potential. J. Glaucoma, 8: 140-148.

Leguire, L.E., Rogers, G.L., Bremer, D.L., Walson, P.D. and McGregor, M.L. (1993) Levodopa/carbidopa for childhood amblyopia. Invest. Ophthalmol. Vis. Sci., 34: 3090-3095.

Maffei, L. and Fiorentini, A. (1982) Electroretinographic responses to alternating gratings in the cat. Exp. Brain Res., 48: 327-334.

Martus, P., Korth, M., Horn, F., Junemann, A., Wisse, M. and Jonas, J.B. (1998) A multivariate sensory model in glaucoma diagnosis. Invest. Ophthalmol. Vis. Sci., 39: 1567-1574.

Parisi, V. (1997) Neural conduction in the visual pathways in ocular hypertension and glaucoma. Graefes. Arch. Clin. Exp. Ophthalmol., 235: 136-142.

Parisi, V. (2001) Impaired visual function in glaucoma. Clin. Neurophysiol., 112: 351-358.

Parisi, V. (2003) Correlation between morphological and functional retinal impairment in patients affected by ocular hypertension, glaucoma, demyelinating optic neuritis and Alzheimer's disease. Semin. Ophthalmol., 18: 50-57.

Parisi, V. (2005) Electrophysiological assessment of glaucomatous visual dysfunction during treatment with cytidine- 5 diphosphocholine (citicoline): a study of 8 years of follow-up. Doc. Ophthalmol., 110: 91-102.

Parisi, V. and Bucci, M.G. (1992) Visual evoked potentials after photostress in patients with primary open-angle glaucoma and ocular hypertension. Invest. Ophthalmol. Vis. Sci., 33: 436-442.

Parisi, V., Colacino, G., Milazzo, G., Scuderi, A.C. and Manni, G. (1999a) Effects of nicergoline on the retinal and cortical electrophysiological responses in glaucoma patients: a preliminary open study. Pharmacol. Res., 40: 249-255.
Parisi, V., Manni, G., Centofanti, M., Gandolfi, S.A., Olzi, D. and Bucci, M.G. (2001) Correlation between optical coherence tomography, pattern electroretinogram, and visual evoked potentials in open-angle glaucoma patients. Ophthalmology, 108: 905-912.

Parisi, V., Manni, G., Colacino, G. and Bucci, M.G. (1999b) Cytidine-5'-diphosphocholine (citicoline) improves retinal and cortical responses in patients with glaucoma. Ophthalmology, 106: 1126-1134.

Parisi, V., Manni, G., Gandolfi, S.A., Centofanti, M., Colacino, G. and Bucci, M.G. (1999c) Visual function correlates with nerve fiber layer thickness in eyes affected by ocular hypertension. Invest. Ophthalmol. Vis. Sci., 40: 1828-1833.

Parisi, V., Manni, G., Spadaro, M., Colacino, G., Restuccia, R., Marchi, S., Bucci, M.G. and Pierelli, F. (1999d) Correlation between morphological and functional retinal impairment in multiple sclerosis patients. Invest. Ophthalmol. Vis. Sci., 40: 2520-2527.

Parisi, V., Miglior, S., Manni, G., Centofanti, M. and Bucci, M.G. (2006) Clinical ability of pattern electroretinograms and visual evoked potentials in detecting visual dysfunction in ocular hypertension and glaucoma. Ophthalmology, 113: 216-228

Parisi, V., Pernini, C., Guinetti, C., Neuschuler, R. and Bucci, M.G. (1997) Electrophysiological assessment of visual pathways in glaucoma. Eur. J. Ophthalmol., 7: 229-235.

Pecori-Giraldi, J., Virno, M., Covelli, G., Grechi, G. and De Gregorio, F. (1989) Therapeutic value of citicoline in the treatment of glaucoma (computerized and automated perimetric investigation). Int. Ophthalmol., 13: 109-112.

Porciatti, V., Falsini, B., Brunori, S., Colotto, A. and Moretti, G. (1987) Pattern electroretinogram as a function of spatial frequency in ocular hypertension and early glaucoma. Doc. Ophthalmol., 65: 349-355.

Porciatti, V., Schiavi, C., Benedetti, P., Baldi, A. and Campos, E.C. (1998) Cytidine-5'-diphosphocholine improves visual acuity, contrast sensitivity and visually-evoked potentials of amblyopic subjects. Curr. Eye Res., 17: 141-148.

Rejdak, R., Toczolowski, J., Kurkowski, J., Kaminski, M.L., Rejdak, K., Stelmasiak, Z. and Grieb, P. (2003) Oral citicoline treatment improves visual pathway function in glaucoma. Med. Sci. Monit., 9: PI24-PI28.

Salgarello, T., Colotto, A., Falsini, B., Buzzonetti, L., Cesari, L., Iarossi, G. and Scullica, L. (1999) Correlation of pattern electroretinogram with optic disc cup shape in ocular hypertension. Invest. Ophthalmol. Vis. Sci., 40: 1989-1997.

Secades, J.J. and Frontera, G. (1995) CDP-choline: pharmacological and clinical review. Methods Find Exp. Clin. Pharmacol., 17(Suppl B): 1-54.

Serra, I., Alberghina, M., Viola, M., Ristretta, A. and Giuffrida, A.M. (1981) Effect of CDP-choline on the biosynthesis of nucleic acids and proteins in brain regions during hypoxia. Neurochem. Res., 6: 607-618.

Vaegan, G.S.L., Goldberg, I., Buckland, L. and Hollows, F.C. (1995) Flash and pattern electroretinogram changes with optic atrophy and glaucoma. Exp. Eye Res., 60: 697-706. 
Ventura, L.M. and Porciatti, V. (2005) Restoration of retinal ganglion cell function in early glaucoma after intraocular pressure reduction: a pilot study. Ophthalmology, 112: 20-27.

Ventura, L.M., Porciatti, V., Ishida, K., Feuer, W.J. and Parrish, R.K., 2nd (2005) Pattern electroretinogram abnormality and glaucoma. Ophthalmology, 112: 10-19.

Virno, M., Pecori-Giraldi, J., Liguori, A. and De Gregorio, F. (2000) The protective effect of citicoline on the progression of the perimetric defects in glaucomatous patients (perimetric study with a 10-year follow-up). Acta Ophthalmol. Scand., 232(Suppl): 56-57.

Watanabe, I., Iijima, H. and Tsukahara, S. (1989) The pattern electroretinogram in glaucoma: an evaluation by relative amplitude from the Bjerrum area. Br. J. Ophthalmol., 73: 131-135.

Weber, A.J., Chen, H., Hubbard, W.C. and Kaufman, P.L. (2000) Experimental glaucoma and cell size, density, and number in the primate lateral geniculate nucleus. Invest. Ophthalmol. Vis. Sci., 41: 1370-1379.

Weiss, G.B. (1995) Metabolism and actions of CDP-choline as an endogenous compound and administered exogenously as citicoline. Life Sci., 56: 637-660.

Yucel, Y.H., Zhang, Q., Gupta, N., Kaufman, P.L. and Weinreb, R.N. (2000) Loss of neurons in magnocellular and parvocellular layers of the lateral geniculate nucleus in glaucoma. Arch. Ophthalmol., 118: 378-384.

Yucel, Y.H., Zhang, Q., Weinreb, R.N., Kaufman, P.L. and Gupta, N. (2003) Effects of retinal ganglion cell loss on magno-, parvo-, koniocellular pathways in the lateral geniculate nucleus and visual cortex in glaucoma. Prog. Retin. Eye Res., 22: 465-481.

Zappia V., Kennedy P., Nilsson B.I. and Galletti P. (Eds.), (1985). Novel Biochemical, Pharmacological and Clinical Aspects of Cytidine-diphosphocholine. Elsevier, New York. 